$01 ; 14$

\title{
Определение степени синхронности перемежающейся фазовой синхронизации по данным электроэнцефалограмм человека
}

\author{
(ㄱ А.Д. Колоскова, О.И. Москаленко
}

Саратовский национальный исследовательский государственный университет им. Н.Г. Чернышевского

๙E-mail: o.i.moskalenko@gmail.com

Поступило в Редакцию 9 января 2017г.

Обнаружена перемежающаяся фазовая синхронизация при развитии эпилептической активности человека. Показано наличие фаз синхронного поведения как во время пик-волновых разрядов, так и в областях фоновой активности головного мозга. Определена степень синхронности режима перемежающейся фазовой синхронизации в обоих случаях и установлено, что пик-волновые разряды характеризуются более высокой степенью синхронности по сравнению с участками фоновой активности головного мозга. Для оценки степени синхронизации предложена модификация метода оценки величины нулевого условного показателя Ляпунова по временному ряду.

DOI: 10.21883/PJTF.2017.10.44627.16701

В реальных физиологических системах часто встречается такое явление, как фазовая хаотическая синхронизация $[1,2]$. Данный режим характерен как для неавтономных, так и для связанных хаотических систем и означает установление между их состояниями захвата фаз при отсутствии связи между их амплитудами [3,4].

На границе фазовой хаотической синхронизации возникает перемежающаяся фазовая синхронизация [5,6]. Это режим, при котором захват фаз происходит только в определенные моменты времени. Такие интервалы называются ламинарными (синхронными) участками поведения. Синхронные участки прерываются турбулентными всплесками, характеризующими кратковременный рост разности фаз. Такой тип поведения наблюдается также в неавтономных периодических осцилляторах, находящихся под внешним шумовым воздействием, а также 
в реальных нейрофизиологических системах, например при развитии эпилептической активности у человека и животных [2,7].

Понятия „синхронизация“ и „захват фаз“ тесным образом связаны с поведением так называемого условного нулевого показателя Ляпунова [8]. В частности, известно, что переход нулевого условного показателя Ляпунова в область отрицательных значений в неавтономных системах (демонстрирующих периодическую динамику) в присутствии шума и связанных хаотических системах предшествует установлению режима фазовой синхронизации. При этом стоит отметить, что разница между критическими значениями параметра связи, соответствующими порогу фазовой синхронизации и моменту перехода показателя Ляпунова в отрицательную область, может быть достаточно большой. Иными словами, условный нулевой показатель Ляпунова оказывается отрицательным задолго до возникновения режима фазовой синхронизации, а следовательно, его величина может быть рассмотрена как степень синхронизма перемежающейся фазовой синхронизации, имеющей место на границе возникновения синхронного режима [8-10].

Без особого труда можно найти данную величину, если оператор эволюции системы задан в явном виде [11]. Для некоторых случаев разработаны методы, которые решают поставленную задачу даже в том случае, когда оператор эволюции неизвестен (по временной реализации системы), однако все они обладают недостатками (в первую очередь высокой чувствительностью к шумам и ошибкам), для преодоления которых предлагаются новые методы и алгоритмы [12,13].

Целью настоящей работы является разработка нового, улучшенного метода для расчета условного нулевого показателя Ляпунова взаимодействующих систем по временному ряду и его применение для оценки степени режима перемежающейся фазовой синхронизации реальной нейрофизиологической системы. Наличие такого метода позволит в перспективе более эффективно осуществлять обработку данных и диагностировать наличие и степень синхронного режима по нейрофизиологическим данным, что, в свою очередь, может быть полезно при обнаружении/использовании данного эффекта в практических приложениях, в частности при осуществлении медицинской диагностики. С другой стороны, данный метод может быть рассмотрен как самостоятельный инструмент для проведения исследований: метод может быть применен к достаточно коротким экспериментальным временным рядам любой природы, позволяя продиагностировать наличие/степень синхронности

Письма в ЖТФ, 2017, том 43, вып. 10 


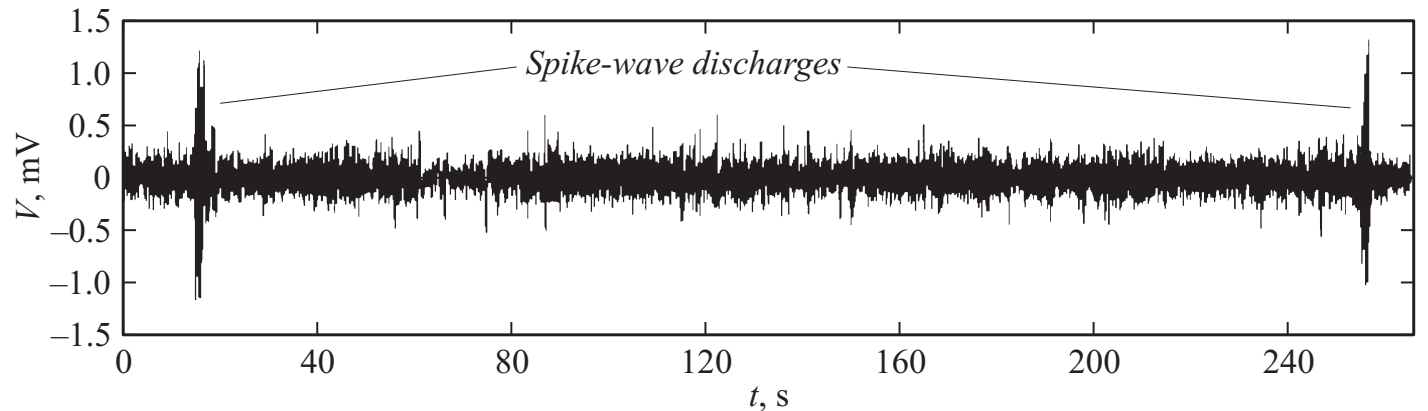

Рис. 1. Сигнал ЭЭГ, снятый с отдела С3 головного мозга человека. 
наблюдаемого режима, в то время как другими методами сделать это не представляется возможным.

В качестве исследуемых сигналов были выбраны реальные экспериментальные данные нейрофизиологической природы - сигналы электроэнцефалограмм (ЭЭГ), снятые с различных отделов головного мозга человека, страдающего эпилепсией. Один из таких сигналов приведен на рис. $1^{1}$. Эпилептические ЭЭГ представляют собой перемежающиеся временные реализации, содержащие в себе пик-волновые разряды и чередующиеся с ними участки фоновой активности головного мозга. Известно, что пик-волновые разряды характеризуются высокой степенью синхронизма $[7,14]$. В то же самое время, как показали проведенные исследования, на участках фоновой активности также возможно существование фаз синхронного поведения.

Рис. 2 иллюстрирует зависимости разности фаз $x(n)=\varphi_{1}-\varphi_{2}$ сигналов, снятых с отделов $\mathrm{C} 3$ и $\mathrm{Cz}$ головного мозга человека (расположение электродов показано в работе [15] на рис. 24). На рисунках показаны только участки синхронного поведения. Рис. 2, $а$ соответствует пик-волновым разрядам, рис. $2, b-$ синхронным участкам фоновой активности головного мозга. Фазы $\varphi_{1,2}$ рассматриваемых сигналов вводились в рассмотрение при помощи непрерывного вейвлетного преобразования с материнским вейвлетом Морле $[7,16]$.

Для синхронных участков каждого вида динамики был применен следующий метод оценки величины условного нулевого показателя Ляпунова.

По аналогии с работами $[9,17]$ расчет показателя Ляпунова осуществлялся по формуле

$$
\Lambda=\int_{x_{1}}^{x_{2}} \rho(x) \ln |1+2 \Omega x| d x,
$$

где $\rho(x)$ - плотность распределения вероятности для значений $x$. Плотность вероятности $\rho(x)$ рассчитывалась численно и аппроксимировалась аналитической закономерностью

$$
\rho(x)=A \exp \left[-\frac{2}{D}\left(\varepsilon x-\frac{\Omega x^{3}}{3}\right)\right]
$$

${ }^{1}$ Сигналы ЭЭГ, соответствующие другим отделам головного мозга, аналогичны рис. 1.

Письма в ЖТФ, 2017, том 43, вып. 10 

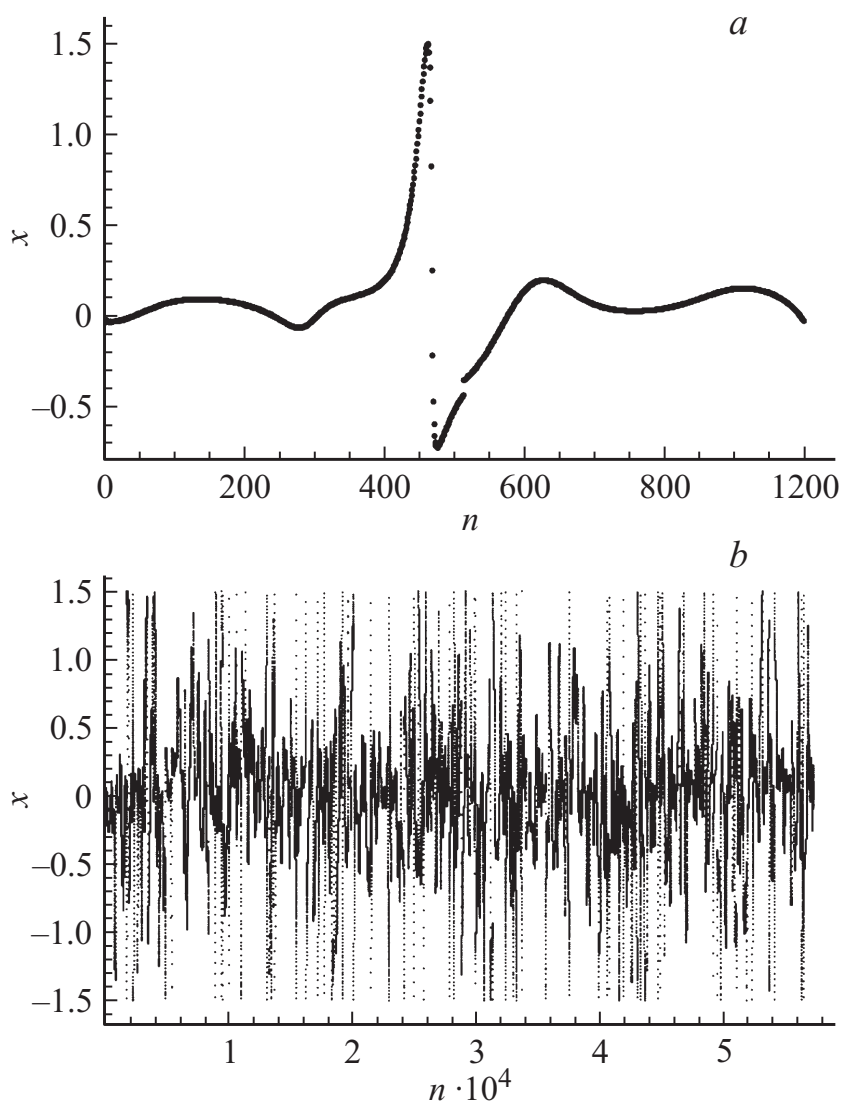

Рис. 2. Временные зависимости разности фаз $x(n)$ между сигналами, снятыми с отделов $\mathrm{C} 3$ и $\mathrm{Cz}$ головного мозга человека, в течение пик-волновых разрядов $(a)$ и синхронных участков фоновой активности головного мозга человека $(b)(n-$ дискретное время). 
(где $A-$ коэффициент нормировки, определяемый из условия

$$
\int_{x_{1}}^{x_{2}} \rho(x) d x=1,
$$

$D-$ интенсивность шума, $\varepsilon$ и $\Omega-$ управляющие параметры), характерной для закритической области значений управляющего параметра $\varepsilon$, соответствующей режиму перемежающейся фазовой синхронизации $[8,9]$.

Поиск параметров аппроксимации осуществлялся следующим образом. Для нахождения параметра $D$ было сделано предположение о том, что распределение плотности вероятности $\rho(x)$ визуально напоминает гауссово распределение плотности вероятности, которое в упрощенном виде можно записать как

$$
\rho_{G}(x)=A_{G} \exp \left[-2 B(x-K)^{2}\right],
$$

где $K$ и $B-$ аналоги математического ожидания и дисперсии, $A_{G}-$ нормировочный множитель. Параметр $K$ соответствует максимуму распределения (4), а следовательно, оказывается связанным с параметрами $\Omega$ и $\varepsilon$ распределения (2) следующим образом:

$$
K=-\sqrt{\frac{\varepsilon}{\Omega}} .
$$

Раскладывая правые части (2) и (4) в ряд Тейлора в точке (5), ограничившись вторым порядком малости и сравнивая коэффициенты произведенных разложений при равных степенях, можно найти связь между параметрами $B$ и $D$ :

$$
D=\frac{\sqrt{\varepsilon \Omega}}{B} .
$$

Параметры $A_{G}$ и $K$ могут быть найдены из сопоставления максимумов численно полученного распределения вероятности и его аппроксимации закономерностью (4); параметр $B$ находится при помощи метода наименьших квадратов. Тогда связь между параметрами $D, \varepsilon$ и $\Omega$ определяется соотношением (6), где параметр $B$ является известным, что делает возможным поиск остальных параметров аппроксимации:

Письма в ЖТФ, 2017, том 43, вып. 10 

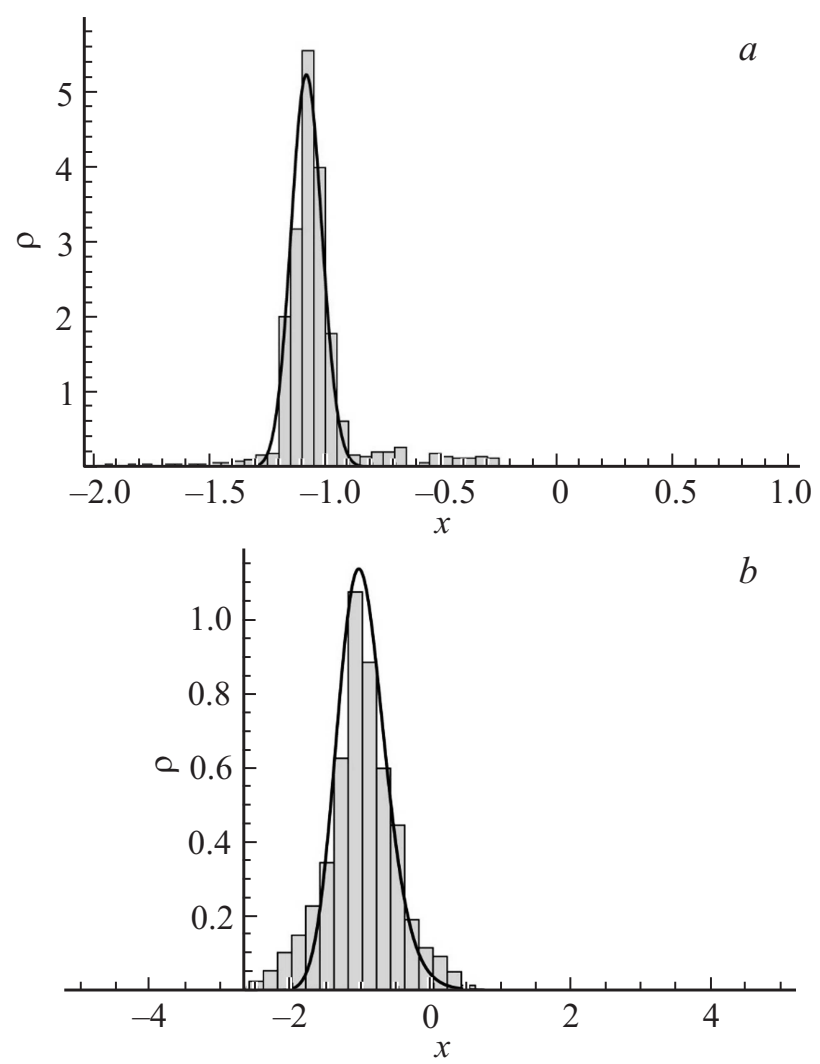

Рис. 3. Распределения плотности вероятности $\rho(x)$, полученные для пикволновых разрядов $(a)$ и для участков фоновой активности головного мозга человека $(b)$ (гистограммы), и их аппроксимации закономерностью (2). 
связь между параметрами $A, \varepsilon$ и $\Omega$ находится также из сопоставления максимумов аналитически (распределение (2)) и численно полученных распределений, а параметр $\Omega$ находится при помощи метода наименьших квадратов. Параметры $x_{1}$ и $x_{2}$ в формуле (1) определяются эмпирически из вида $\rho(x)$.

Предложенный метод применен как к пик-волновым разрядам, так и к участкам фоновой активности головного мозга человека. Полученные распределения плотности вероятности разности фаз и их аппроксимация закономерностью (2) приведены на рис. 3, $a$ (пик-волновые разряды) и $3, b$ (синхронные участки фоновой активности) соответственно. В первом случае были получены параметры аппроксимации $B \approx 56.70$, $D \approx 0.182, \varepsilon \approx 0.468, A \approx 1.917 \cdot 10^{-38}, \Omega \approx 0.4$, во втором случае $B \approx 2.30, D \approx 0.182, \varepsilon \approx 0.441, A \approx 0.384, \Omega \approx 0.4$. Показатели Ляпунова в обоих случаях оказываются отрицательными, а их отношение друг к другу оказывается равным $\Lambda_{1} / \Lambda_{2} \approx 1.12$, что говорит о более высокой степени синхронизма пик-волновых разрядов по сравнению с участками фоновой активности головного мозга человека.

Таким образом, в настоящей работе предложен метод оценки степени синхронности режима перемежающейся фазовой синхронизации по временному ряду, основанный на вычислении условного нулевого показателя Ляпунова. Метод применен к нейрофизиологическим данным - электроэнцефалограммам человека, страдающего эпилепсией. Показано, что пик-волновые разряды характеризуются более высокой степенью синхронизма по сравнению с участками фоновой активности головного мозга.

Авторы благодарны А.А. Короновскому, А.Е. Храмову и М.О. Журавлеву за предоставленные данные и помощь в их обработке. Работа выполнена при поддержке Совета по грантам Президента РФ для поддержки молодых ученых (МК-4574.2016.2).

\section{Список литературы}

[1] Janson N.B., Balanov A.G., Anishchenko V.S., McClintock P.V.E. // Phys. Rev. Lett. 2001. V. 86 (9). P. 1749-1752.

[2] Bob P., Palus M., Susta M., Glaslova K. // Neurosci. Lett. 2008. V. 447. P. 7377.

[3] Анищенко В.С., Постнов Д.Э. // Письма в ЖТФ. 1988. Т. 14. В. 6. С. 569.

Письма в ЖТФ, 2017, том 43, вып. 10 
[4] Rosenblum M.G., Pikovsky A.S., Kurths J. // Phys. Rev. Lett. 1996. V. 76 (11). P. 1804-1807.

[5] Pikovsky A.S., Osipov G.V., Rosenblum M.G. et al. // Phys. Rev. Lett. 1997. V. 79 (1). P. 47-50.

[6] Hramov A.E., Koronovskii A.A., Kurovskaya M.K., Boccaletti S. // Phys. Rev. Lett. 2006. V. 97. P. 114101.

[7] Hramov A.E., Koronovskii A.A., Midzyanovskaya I.S. et al. // Chaos. 2006. V. 16. P. 043111.

[8] Hramov A.E., Koronovskii A.A., Kurovskaya M.K. // Phys. Rev. E. 2008. V. 78. P. 036212.

[9] Moskalenko O.I., Koronovskii A.A., Hramov A.E. // Phys. Rev. E. 2015. V. 92. P. 012913.

[10] Короновский А.А., Куровская М.К., Храмов А.Е., Шурыгина С.А. // ЖТФ. 2009. T. 79. B. 10. C. 1-9.

[11] Benettin G., Galgani L., Giorgilli A., Strelcyn J.-M. // Meccanica. 1980. V. 15. P. 9-30.

[12] Wolf A., Swift J., Swinney H.L., Vastano J. // Physica D. 1985. V. 16. P. 285.

[13] Москаленко О.И., Павлов А.С. // Письма в ЖТФ. 2014. Т. 40. В. 12. С. 66-72.

[14] Hramov A.E., Koronovskii A.A., Makarov V.A. et al. Wavelets in Neuroscience. Heidelberg, New York, Dordrecht, London: Springer, 2015.

[15] Павлов А.Н., Храмов А.Е., Короновский А.А. et al. // УФН. 2012. Т. 182. B. 9. C. $905-939$.

[16] Hramov A.E., Koronovskii A.A. // Chaos. 2004. V. 14 (3). P. 603-610.

[17] Москаленко О.И., Короновский А.А., Храмов А.Е., Журавлев М.О. // Письма в ЖЭТФ. 2016. Т. 103 (8). С. 606-610. 